www.jmscr.igmpublication.org

Impact Factor (SJIF): 6.379

Index Copernicus Value: 71.58

ISSN (e)-2347-176x ISSN (p) 2455-0450

crossref DOI: https://dx.doi.org/10.18535/jmscr/v6i6.24

Journal Of Medical Science And Clinical Research

IGM Publication

An Official Publication of IGM Publication

\title{
Comparison between Mulligan Traction Leg Raise versus Slumps Stretching on Pain, Passive Leg Raise, and Functional Disability in Lumbar Radiculopathy
}

\author{
Authors \\ Swati Mishra $^{1^{*}}$, Dr Bindu Sarfare $(\text { PT })^{2}$, Dr Snehal Ghodey $(\text { PT })^{3}$ \\ ${ }^{1}$ BPTH Intern, MAEER'S Physiotherapy College, Talegaon Dabhade \\ ${ }^{2}$ Associate professor, MAEER'S Physiotherapy College, Talegaon Dabhade \\ ${ }^{3}$ Principal, MAEER'S Physiotherapy College, Talegaon Dabhade \\ *Corresponding Author \\ Swati Mishra \\ Email: swati123.2010@gmail.com
}

\begin{abstract}
Lumbar spine disorders rank 5th among the diseases categories with 37\% of cost a direct case of physical therapy services. So the intervention requires a beneficial, safer, and cost effective treatment. Conservative treatment for sciatica aims at pain reduction but improving the range of SLR has a beneficial effect in restoring normal movement and reduces the impairments due to low back dysfunction. The purpose of the study is compare the effectiveness of mulligan traction leg raise and slumps stretching with conventional therapy so as to determine which technique is better in treating the dysfunction due to lumbar radiculopathy. An experimental study was carried on 40 lumbar radiculopathy patients by simple random sampling. They were randomly assigned into 2 groups of 20 each, Group A was given mulligan traction leg raise and Group $B$ was given slumps stretching with conventional treatment in both the groups for 6 consecutive days. Outcome measures were NRS, passive SLR, oswestry disability index. Result concluded that Mulligan traction leg raise and slumps stretching both are equally effective in reducing pain and functional disability but Slumps stretching is more effective neural mobilization in increasing SLR angle than mulligan traction leg raise.
\end{abstract}

Keywords: Lumbar Radiculopathy, Mulligan Traction Leg Raise, Slumps Stretching.

\section{Introduction}

According to sharma SC, Singh R, Sharma AK, Mittal R: India has an incidence of low back pain (LBP) around $23.09 \%$ and a lifetime prevalence of $60-80 \%$. Onset of LBP is more in population of 30-50 years, where men and women show equal affection. Population under 45 years of age are most commonly affected. ${ }^{1}$ When there is exceed in the normal range of movement it is defined as neural tension. ${ }^{3}$ Lumbar disc syndrome causes abnormal physiological or mechanical response on the structures of the nervous system leading to sciatica. ${ }^{3}$ Sciatica is leg pain in the distribution of one or more lumbosacral nerve roots, irrespective of neurological deficit after second decade of life there intra-discal changes resulting in necrosis, 
sequestration of the annulus fibrosis. In the later stages even a minor strain may cause either internal damage with eccentric displacement of nucleus pulpous or external derangement, leading to burst of nucleus pulposus out through the annulus fibrosis, usually in postero-lateral direction. ${ }^{1}$ Many musculoskeletal pathomehanics which may cause neural consequences are Sacoiliac joint dysfunction, Piriformis syndrome, nerve entrapment due to spasm or severe tightness of the piriformis, Compression of the adjacent sciatica nerve caused by edema, inflammation and hematoma formed around the affected tendon., Hallmark symptoms is pain and it may vary ${ }^{5}$.Iirritation of sciatica nerve cause pain, which can be continuous or intermittent, it can be aggravated by certain movements like coughing, sneezing, sitting, bending, prolonged standing or rising from sitting position. ${ }^{5}$ Pain is located in the rear or back, buttock, knee, and leg ${ }^{5}$. Unilateral radiation of pain can result in reduced power, diminished reflexes and sensation in the nerve roots. ${ }^{5}$ Gait dysfunction(toe walking, foot drop, knee buckling), paresthesias, dysaesthesias are frequently the neurological symptoms (e.g., burning or tingling in the leg, pins and needles in the feet and toes). ${ }^{5}$ Neural mobilization is used for altered neurodynamic or altered neural tension. It aims to restore the relative mobility of the neural tissue and the surrounding mechanical interface, reducing intrinsic pressure and regaining optimum physiological function. ${ }^{6}$ In spite of the knowledge of such benefits little work is available in the, form of RCT'S that can give definitive evidence of the benefits of using neurodynamics techniques for the back pain population. A recent systematic review identified only 10 RCT'S that discussed the therapeutic effect of neural mobilization. ${ }^{6}$. Neural mobilization techniques are used in the instance of altered neurodynamic or altered neural tension. It aims to restore the relative mobility of the neural tissue and the surrounding mechanical interface, reducing intrinsic pressure and regaining optimum physiological function. ${ }^{6}$ In spite of the knowledge of such benefits little work is available in the, form of RCT'S that can give definitive evidence of the benefits of using neurodynamics techniques for the back pain population. A recent systematic review identified only 10 RCT'S that discussed the therapeutic effect of neural mobilization. ${ }^{6}$.

Slumps stretching: The slumps test can be used as a treatment technique when the canal structures are moved through large pain free amplitude or to stretch the structures strongly. ${ }^{7}$

Mulligan traction leg raise: The traction leg raise techniques are painless intervention which are said to have immediate benefits in patients having low back pain with limited hip flexion range. ${ }^{8}$ Conservative treatment for sciatica is primarily aimed at pain reduction but improving the range of SLR has a beneficial effect in restoring normal movement and reducing the degree of impairment due to low back dysfunction. ${ }^{9}$.There is limited research about the effectiveness of mulligan traction leg raise and slumps stretching on symptomatic patients. The role of the physiotherapist in symptomatic patients is to relive the pain at the earliest and also reduce their functional disability. Both the techniques have differently proven to have immediate effects. Hence the study is to compare the technique having benefits for faster relief of pain, improve in ROM of SLR and functional disability.

\section{Materials and Method}

55 Patients diagnosed with lumbar radiculopathy were taken and divided into two groups by random sampling method 26 in mulligan traction leg raise and 29 in slumps stretching group, but during the study there were 15 drop out, out of which each group had 20 subjects. It was an experimental study carried out for 6 consecutive days.

Materials required are pen, paper, consent form, numerical rating scale, goniometer, and oswestry disability index scale.

\section{Inclusion and exclusion criteria}

Participants were included in the study if they meet following criteria (1) pre diagnosed with 
lumbar radiculopathy (2) age group of 18-60 (3) SLR test positive (4) NRS between 2-6(5) All subjects with unilateral or bilateral radiation of pain in sciatic nerve distribution (6) Both male and female (7) Oswestry disability index more than $10 \%$.(8) participants willing to participate in the study ${ }^{3}$.

Participants were excluded if: (1) History of spinal surgery in previous 6 months (2)Knee and ankle pathology causing limitation of movement (3)Clinical conditions such as over sensitive skin, patient with cardiac pacemaker, pregnancy etc, where TENS is contraindicated (4)Serious spinal condition e.g infection, tumors, osteoporosis, spinal fracture (5) Inability to hold slumps stretching position, reproduction of symptoms on neck flexion part of slumps test (6) Patients with cervicogenic headache, VBI, spinal deformities and ankylosing spondylitis. ${ }^{2}$

\section{Procedure}

Subjects according to the inclusion exclusion criteria were taken and divided in two groups. The allocation was done by random sampling chit method. Group A received TENS, lumbar stabilization exercises and mulligan traction leg raise, similarly Group B received TENS, lumbar stabilization exercises and slumps stretching.

\section{Application of transcutaneous Electrical Nerve}

Stimulation (TENS): Participants were positioned in prone lying and were treated with high frequency, short pulse, high intensity,-burst TENS using pad electrodes for 30 minutes per day per sitting. ${ }^{1}$

\section{Lumbar Stabilization exercises}

It includes static back, static hamstring, static glutei 10 seconds hold with 10 repetitions.

\section{Mulligan traction leg raise}

Mulligan traction leg raise was given in patients in supine position on a low couch or floor. Therapist stands beside the patients affected side with knees bend. Therapist holds distal leg (just proximal to both the malleoli) and reinforce this grip with his/her elbow of the other hand. Therapist applies longitudinal traction along the long axis of the leg and then raises patient leg off the bed to a position just short of painful range. While maintaining this traction, therapist simultaneously extends his knees to provide flexion (SLR) on the affected side. Traction is then sustained and leg is raised as far as possible, provided there is no pain. External rotation and/or abduction at the hip can be added, if patient complains of pain. Hold the new available end range for 10 seconds, do not relive the traction till the leg returns to the starting position. Repeat this maneuver 3 times (only if pain free, and the therapist is able to increase the range of motion. $)^{9}$

Fig No. 1

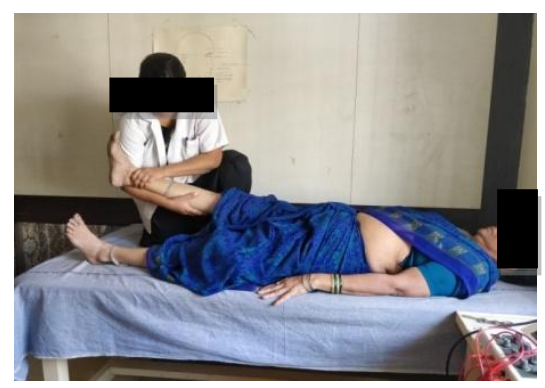

\section{Slumps stretching}

This technique was performed with patient in sitting position on the couch or bed with bilateral feet against the wall. Therapist applied over pressure with one hand to the cervical spine flexion and with other hand to maintain knee in extension to the point_where the patient's pain were reproduced. Position was held for 30 seconds. 3-5 repetitions of stretches were performed in each session based on patients response. Patients were excluded in case of adverse respons. Six consecutive days of treatment was given. After the session, final readings of all the outcome measure were taken and the subjects was discharged from therapy. ${ }^{3}$

Fig No. Il

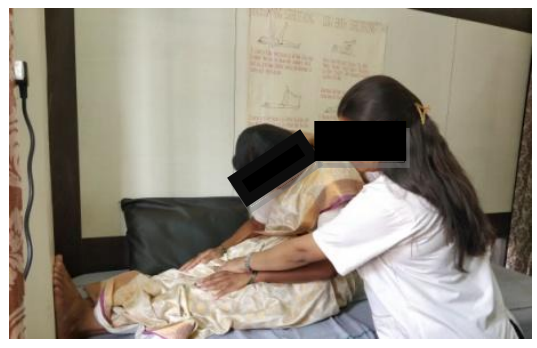




\section{Results}

\section{Demographic Data}

40 Subjects were included in the study in which 26 were female and 14 male were included.

Fig No.lll

Graph Showing Distribution of Male and Female

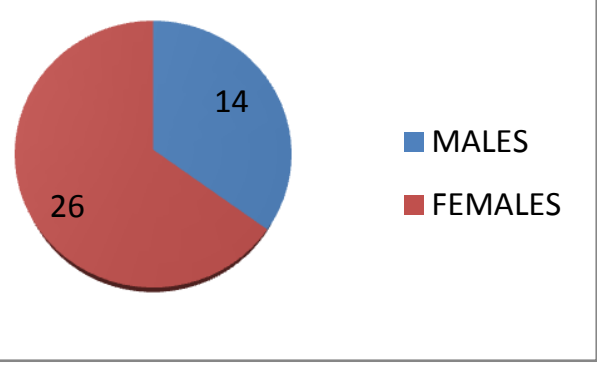

Fig No. IV

Graph Showing Comparison of inter Group Mean of NRS

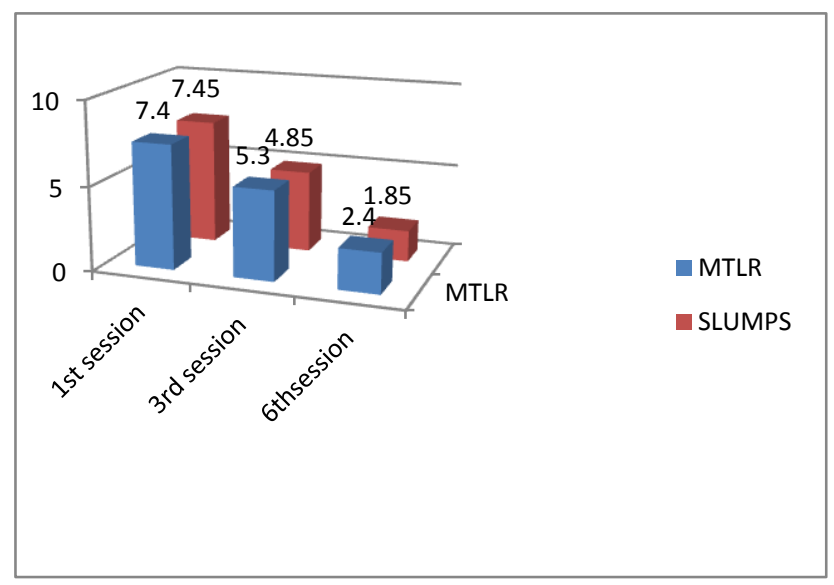

Table no. 1

\begin{tabular}{|l|c|c|c|c|c|}
\hline \multicolumn{6}{|c|}{ Comparison of mean of NRS between MTLR and SLUMPS } \\
\hline SR NO. & GROUP & $\mathbf{N}$ & \multicolumn{3}{|c|}{ MEAN AND SD } \\
\hline & & & $\begin{array}{c}\mathbf{1}^{\text {ST }} \\
\text { SESSION }\end{array}$ & $\begin{array}{c}\text { 3rd } \\
\text { SESSION }\end{array}$ & $\begin{array}{c}\mathbf{6}^{\text {th }} \\
\text { SESSION }\end{array}$ \\
\hline$\underline{\mathbf{1}}$ & MTLR & $\mathbf{2 0}$ & 7.4 & 5.3 & 2.4 \\
\hline & & & 1.391 & 1.380 & 1.569 \\
\hline$\underline{\mathbf{2}}$ & Slumps & $\mathbf{2 0}$ & 7.45 & 4.85 & 1.85 \\
\hline & & & 1.05 & 1.424 & 1.49 \\
\hline & P Value & & 0.9783 & 0.2894 & 0.3086 \\
\hline
\end{tabular}

Fig No. V

Graph showing comparison of inter group mean of passive SLR

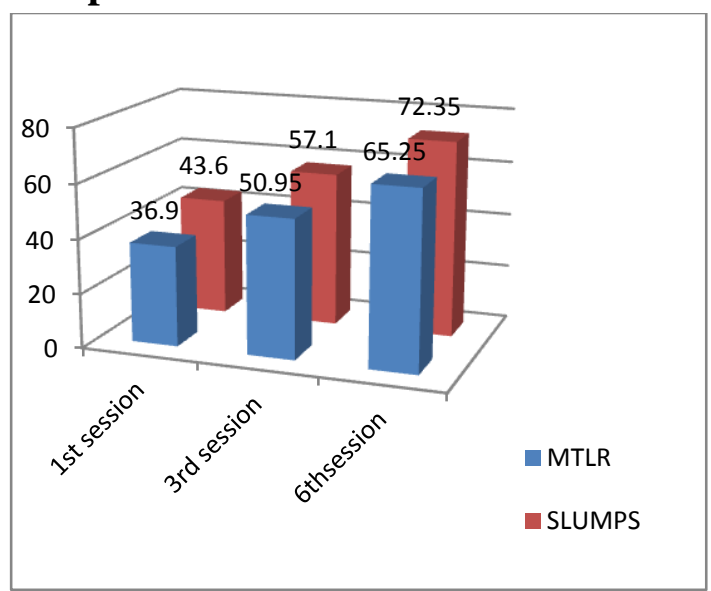

Table no.ll

\begin{tabular}{|c|c|c|c|c|c|}
\hline \multicolumn{6}{|c|}{$\begin{array}{l}\text { Comparison of mean of passive ROM of SLR between MTLR and } \\
\text { SLUMPS }\end{array}$} \\
\hline SR NO. & GROUP & $\mathbf{N}$ & \multicolumn{3}{|c|}{$\begin{array}{l}\text { MEAN AND SD } \\
\end{array}$} \\
\hline & & & $\begin{array}{c}\text { 1st } \\
\text { session }\end{array}$ & $\begin{array}{c}3^{\text {rd }} \\
\text { session }\end{array}$ & $\begin{array}{c}6^{\text {th }} \\
\text { session }\end{array}$ \\
\hline \multirow[t]{2}{*}{1} & MTLR & 20 & 36.95 & 50.95 & 65.2 \\
\hline & & & 12.41 & 12.56 & 9.60 \\
\hline \multirow[t]{3}{*}{2} & SLUMPS & 20 & 43.6 & 57.1 & 72.3 \\
\hline & & & 13.65 & 11.83 & 8.26 \\
\hline & P Value & & 0.112 & 0.165 & 0.01 \\
\hline
\end{tabular}

Fig no. Vl

Graph showing comparision of inter group mean of oswestry disability index

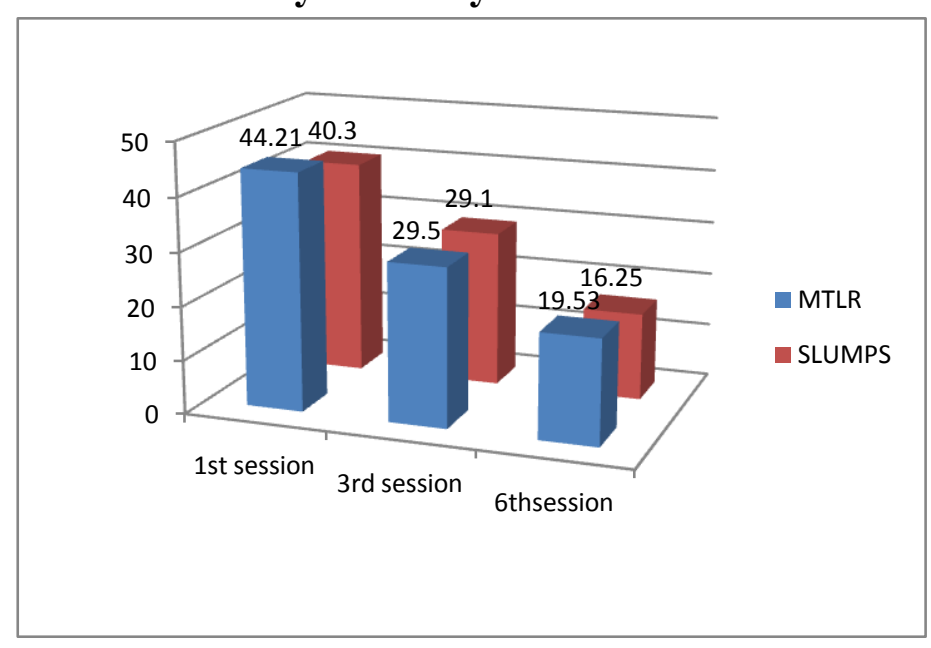


Table no. III

\begin{tabular}{|c|c|c|c|c|c|}
\hline \multicolumn{6}{|c|}{$\begin{array}{l}\text { Comparison of mean of oswestry disability between } \\
\text { MTLR and SLUMPS }\end{array}$} \\
\hline $\begin{array}{l}\text { SR. } \\
\text { NO }\end{array}$ & GROUP & $\mathbf{N}$ & \multicolumn{3}{|c|}{ MEAN AND SD } \\
\hline & & & $\begin{array}{l}\mathbf{1}^{\mathrm{ST}} \\
\text { Session }\end{array}$ & $\begin{array}{l}3^{\mathrm{RD}} \\
\text { Session }\end{array}$ & $\begin{array}{l}6^{\mathrm{TH}} \\
\text { Session }\end{array}$ \\
\hline \multirow[t]{2}{*}{1} & MTLR & 20 & 44.21 & 29.5 & 19.53 \\
\hline & & & 10.71 & 6.93 & 7.83 \\
\hline \multirow[t]{3}{*}{2} & Slumps & 20 & 40.3 & 29.1 & 16.25 \\
\hline & & & 11.79 & 11.61 & 7.23 \\
\hline & P Value & & 0.3506 & 0.473 & 0.167 \\
\hline
\end{tabular}

\section{Fig No. Vll}

Comparision between post treatment score of NRS, passive ROM of SLR, oswestry disability index

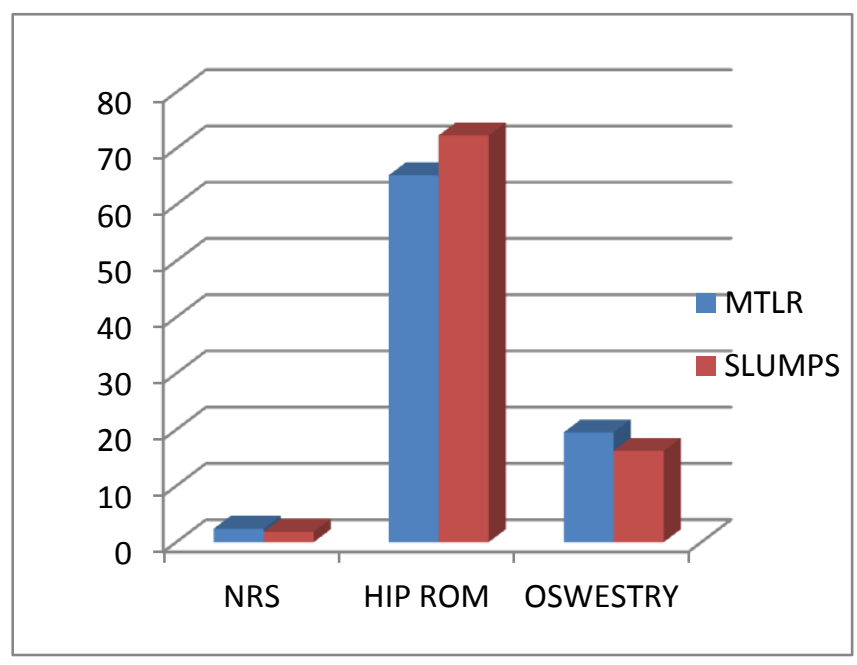

\section{Discussion}

The current study was undertaken to compare the mulligan traction leg raise versus slumps stretching for pain, passive leg raise and functional disability in lumbar radiculopathy. Both the groups were given conventional treatment i.e TENS and lumbar stabilization exercise (static back, static hams, static glutei). Each patient received total six session. In the study NRS ,ROM of SLR, Oswestry disability index were the tools used to evaluate effect of treatment on pain, passive leg raise, functional disability in lumbar radiculopathy patients.

The results showed that statistically the mean post treatment scores of NRS at first session was 7.4 and 7.45 in Group A and Group B respectively given by the $\mathrm{p}$ value 0.9783 using mann whitney, non parametric test which was considered not significant and similarly it was seen in 3rd and 6th session. But post treatment there was significant improvement in the scores of NRS in both the groups i.e at end of sixth session given by $p$ value $<0.0001$ using wilcoxon, non parametric test which is considered extremely significant.

Similarly within the group significant difference was seen in the ROM of SLR shown by $p$ value $<0.0001 \quad$ (extremely significant.)Post treatment Mean was 72.35 and of 65.25 of mulligan and slumps group respectively given by $\mathrm{p}$ value is 0.0168 which is considered quite significant. This shows slumps stretching is better than mulligan traction leg raise in improving ROM of SLR.

Individually post treatment (6th session) both the groups were effective in improving the score of oswestry disability index given by the $p$ value $<0.0001$ which was extremely significant. The mean score of Oswestry disability index at first session was 44.21 and 40.3 in group $\mathrm{A}$ and Group B respectively given by $\mathrm{p}$ value 0.3506 which was considered not significant, similarly in 3rd and 6th session. When we compared post 6th session treatment there was not quite significant improvement in both the groups given by $\mathrm{p}$ value 0.1673 considered not significant.

There was graphically and statistically significant decrease in pain, increase in passive straight leg raise, and decrease in score of oswestry disability index in both the group, but When we compared the two groups no statistical difference was seen for pain and functional disability, only Passive SLR of slumps stretching was considered quite significant than mulligan group. This suggests that, both the techniques are equally effective in reducing pain and improving the functional disability, except slumps stretching increases passive ROM of SLR more than mulligan group in lumbar radiculopathy patients.

Neural mobilization has shown reduce in pain. It is hypothesized, during neural mobilization, which is oscillatory technique like mulligan traction leg raise, it is elongating and shortening the nerve 
which increases intra neural pressure temporarily followed by a relaxation period. ${ }^{10}$ This repeated pumping action may enhances dispersal of local inflammatory products in and around the nerve, alleviating hypoxia and reducing pain. In traction leg raise which would stretch as well as mobilize the nerve did produce pain relief, definitely symptoms were not worsened or provoked by straight leg raise traction. In this technique muscle was elongated to tolerated length and this position was held with muscle in its greatest tolerated length. Traction has shown to improve painfully restricted straight leg raise in previous studies by Larsson and pal. ${ }^{11}$ Also a recent study by $\mathrm{C}$. Beyerlein $^{8}$ found that straight leg raise traction technique significantly improved the SLR angle with the similar explanation for the cause of improvement of range. This strongly supports that mulligan traction leg raise can be used successfully in reducing pain and improving ROM of SLR.

Slumps stretching has been found to be effective in the treatment of low back pain. It has been hypothesized that it decreases the patients pain by depressing the intraneural edema. ${ }^{12}$ Beneuciket $\mathrm{al}^{13}$ found that the neural tensioning techniques resulted in C-fibers mediated hypolgesia. The slumps stretching has also been associated with the inhibitory effects ${ }^{14}$ on the sympathetic nervous system, a stimulation of which affects the capability of the nerve to stretch. ${ }^{15}$ Slumps stretching may also be responsible for reducing scar tissue adhered to the neural tissue and surrounding structures. slumps mobilization has also been found to affect posterior myofascial chain flexibility thus increasing the tibio-tarsal joint angle and finger-floor distance which could be another possible mechanism in the greater improvement of range. ${ }^{16}$

Slumps stretching is still better than mulligan traction leg raise because it can be performed by the patient himself. Also this techniques is simple and less time consuming and cost effective than the conventional treatment.

\section{Conclusion and clinical implication}

Mulligan traction leg raise and slumps stretching both are equally effective in reducing pain and functional disability. Slumps stretching is more effective neural mobilization technique in increasing SLR angle than mulligan traction leg raise. Patient can perform self slumps stretching.

\section{Scope and limitation}

This study could have been done on larger number of sample size to judge the effect of the treatment, more number of session could have been under taken, also there was no follow up.

\section{Acknowledgement}

I would like to thank my parents for their constant love and support.

I am extremely grateful to Dr. SNEHAL GHODEY principal MAEER'S physiotherapy college for being the guiding light.

I am extremely thankful to Dr. BINDU SARFARE, associate professor, for constant guidance.

I also want to extend a deep sense of gratitude to the teachers and my batch mates for their encouragement and support.

Lastly I thank the participants for their cooperation towards completion of my study.

\section{References}

1. Sharma SC, Singh R, Sharma AK, Mittal $\mathrm{R}$ : incidence of low back pain in work age adults in rural north india, medical journal of india 2003; 57(4):145-147.

2. DR. Amrutkuvar H. pawar, DR. Santosh metgud. Comparative effectiveness of mulligan's traction straight leg raise and bent leg raise in low back ache with radiculopathy: A randomized clinical trial. International journal of science and research.Vol.3 issue 10, October (2014)

3. Neha malik, Chitrakataria et al. Comparative effectiveness of straight leg raise and slumps stretching in subjects with adverse neural tension. International 
journal of health and rehabilitation sciences.Vol.1 July (2012).

4. Essential orthopedics; J Maheshwari.

5. Sciatica-causes, clinical presentation, Rolland morris questionnaire, physiopedia.com.

6. Ellis RF, WA. Neural mobilization: A Systemic review of randomized controlled trials with an analysis of therapeutic efficacy. Journal of manual and manipulative therapy. 2008; 16(1):8-22.

7. Vimal V, Sreeaj S R, Mini Mol P: A comparative study between low grade mobilization and slumps stretching in the management of low back pain. International journal of medicine and allied health sciences $|2014|$ Vol 1| Issue 2.

8. Mulligan BR. Manual therapy: NAGS, SNAGS, MWMS, etc $5^{\text {th }}$ edition 2006; 70730.

9. Manual of mulligan concept; Dr Deepak kumar.

10. Brown CL, Gilbert KK, Brismee JM, Sizer PS, Smith MP et al. The effects of neurodynamic mobilization on fluid dispersion with in tibial nerve at the ankle: an unembalmedcaraveric study, Jounal of manual and manipulative therapy, 199(12011): 26-34(9).

11. pal B et al: A controlled trail of lumbar traction in the treatment of back pain and sciatica. British Journal of rheumatology: 1986: 25: 181-183.

12. Clelnda JA, Childs JD, Palmar JA, Eberhart S. slumps stretching in the management of non-radicular low back pain: A Pilot clinical trail. Manual Therapy. 2006; 11:279-286.

13. Beneciuk JM, Bishop MD, Geroge SZ. Effect of upper extremity neural mobilization on the thermal pain sensitivity: a sham-controlled study in symptomatic participants. Journal of orthopaedic and sports physical therapy 39(6):428-438.

14. Clenda J, Durall C, Seoll SA. Effects of slumps long sitting on sudomotor and vasomotor function: a pilot study. Journal of manual and manipulative therapy 2002; 10(2): 67-75.

15. Dworkin M, Kuja J, Bialoszewski D, Slupik A, Kiebzak W. Electromyographic and clinical evaluation ofthe efficacy of neuromobilization in patients with low back pain. Orthopedia traumatologia rebilitacja 2009;2(6);11:164-176

16. Diniz KT, Mironda RM, Riberio CD, Vasconcelos DA, FilhoJEC. Neural mobilization effects in posterior myofascial chain flexibility and in head posture. Neurobiologia 2010; 73(3): 53-57. 